\title{
Regression of "fixed" pulmonary vascular resistance in heart transplant candidates after unloading with ventricular assist devices
}

\author{
Friedhelm Beyersdorf, MD, PhD, Christian Schlensak, MD, PhD, Michael Berchtold-Herz, MD, and \\ Georg Trummer, MD
}

Pulmonary hypertension is a risk factor for early and late mortality after heart transplantation. An increase in pulmonary artery pressure secondary to long-standing elevations in left atrial pressures is observed in $33 \%$ to $72 \%$ of all patients with end-stage heart failure. This increase is disproportionate to the increase in left atrial pressure, thus causing an increased transpulmonary gradient and an increased pulmonary vascular resistance (pulmonary vascular resistance [Wood units] = transpulmonary gradient/cardiac output) over time. All patients with increased pulmonary vascular resistance greater than 3 Wood units or transpulmonary gradients greater than $10 \mathrm{~mm} \mathrm{Hg}$ are subjected to a standardized protocol testing the reversibility of the elevated pulmonary artery pressure by pharmacologic interventions before cardiac transplantation. Pulmonary vascular resistance that cannot be lowered pharmacologically is termed " fixed" pulmonary vascular resistance. It has been shown that ventricular assist devices are able to reduce pulmonary vascular resistance even in patients who are unresponsive to pharmacologic reduction of pulmonary vascular resistance, thus allowing a listing for transplantation after a relatively short period of mechanical support (ie, 3-6 months).

\section{SIGNIFICANCE OF PULMONARY HYPERTENSION IN PATIENTS WITH END-STAGE HEART FAILURE}

Pulmonary hypertension (PHT) is a risk factor for early and late mortality after heart transplantation (HTX) with mortality rates up to $40 \% .^{1,2}$ Moreover, Erickson and associates ${ }^{2}$ stated that right ventricular dysfunction accounts for approximately $50 \%$ of all cardiac complications and $20 \%$ of early mortality after HTX.

In the initial phase of heart failure, increases in pulmonary artery pressure (PAP) occur in proportion to an increase of left atrial pressures that can be determined indirectly by measuring the pulmonary capillary wedge pressure (PCWP). Therefore, in this early stage with initially normal pulmonary vascular resistance (PVR), no elevated transpulmonary

\footnotetext{
From the Department of Cardiovascular Surgery, University Medical Center, AlbertLudwigs-University Freiburg, Freiburg im Breisgau, Germany.

Address for reprints: Friedhelm Beyersdorf, MD, PhD, Department of Cardiovascular

Surgery, University Medical Center, Albert-Ludwigs-University Freiburg, Hugstetter Str 55, D-79106 Freiburg im Breisgau, Germany (E-mail: friedhelm. beyersdorf@uniklinik-freiburg.de).

J Thorac Cardiovasc Surg 2010;140:747-9

0022-5223/\$36.00

Copyright (C) 2010 by The American Association for Thoracic Surgery doi:10.1016/j.jtcvs.2010.05.042
}

gradient $(\mathrm{TPG})$ exists $(\mathrm{TPG}=$ mean $\mathrm{PAP}-\mathrm{PCWP}) .{ }^{3}$ Often PVR is also described in Wood units (WU $=$ TPG/cardiac output).

However, long-standing elevations in left atrial pressures result in an increase in PAP in about $33 \%$ to $72 \%$ of all patients with end-stage heart failure. ${ }^{3,4}$ This increase in PAP is disproportionate to the increase in left atrial pressure (PCWP), thus causing an increased TPG and an increased PVR over time. It may be speculated that the increased incidence of patients with higher PVR evaluated for HTX may be related to the fact that patients can be supported longer with modern medical treatment until they finally need to be listed for HTX.

According to the severity of PHT, 3 groups have been defined: no PHT (PVR $<3$ WU, TPG $<10 \mathrm{~mm} \mathrm{Hg}$ ); mildto-moderate PHT (PVR 3-6 WU, TPG 10-20 mm Hg); and severe PHT (PVR >6 WU, TPG $>20 \mathrm{~mm} \mathrm{Hg}$ ).

All patients with increased PVR greater than $3 \mathrm{WU}$ or TPG greater than $10 \mathrm{~mm} \mathrm{Hg}$ are subjected to a standardized protocol testing the reversibility of the elevated PAP by pharmacologic interventions before HTX (Table 1). Pharmacologic agents tested include oxygen, nitroglycerin, sodium nitroprusside, inhaled nitric oxide, phosphodiesterase inhibitors, urapidil, prostaglandins $\mathrm{E}_{1}$ and $\mathrm{I}_{1}$, and sildenafil.

\section{PHT AND HTX}

Mortality after HTX is increased if pharmacologic interventions are not able to reduce PVR below certain levels. This threshold is reported to be between 2.5 and 3.5 WU or more. ${ }^{5,6}$ A PVR that cannot be reduced by pharmacologic interventions below either 2.5 or $3.5 \mathrm{WU}$ is usually termed "fixed" PVR. The pathophysiology of "fixed" PHT is multifactorial and seems to include vascular remodeling at the capillary level, in situ thrombosis, and vasoconstriction. ${ }^{7}$

The International Society of Heart and Lung Transplantation Guidelines and the Canadian Cardiovascular Society Consensus Conference on Cardiac Transplantation suggest that PAP greater than $50 \mathrm{~mm} \mathrm{Hg}$ systolic, PVR greater than 4 to $5 \mathrm{WU}$, and a TPG greater than 15 to $16 \mathrm{~mm} \mathrm{Hg}$ are at least relative, if not absolute, contraindications to cardiac transplantation. ${ }^{8,9}$ However up to date, there is no reliable hemodynamic threshold known beyond which right ventricular failure after transplant is certain to occur, nor are there values below which right ventricular failure is always avoidable. ${ }^{10}$ Even if PVR can be reduced to less than 2.5 WU by using a vasodilator and systolic blood 
pressure is less than $85 \mathrm{~mm} \mathrm{Hg}$, the patient remains at high risk for right heart failure. ${ }^{8}$

We have evaluated our own recent 140 HTX patients and found in $29 \%$ (40/140 patients) a PVR greater than $2.5 \mathrm{WU}$ and in $5 \%(7 / 140)$ a PVR greater than $3.5 \mathrm{WU}$ despite pharmacologic interventions (Table 1). A similar percentage of patients with fixed PVR $(18 \%)$ was reported by others. ${ }^{6}$ In one third of patients in another series, elevated PAP was refractory to medical treatment. ${ }^{11}$

Previous treatment options for patients with end-stage heart failure and fixed PVR were heterotopic HTX, combined heart-lung transplantation, and right ventricle-sparing transplantation. ${ }^{4}$ Inasmuch as the results of these alternative treatment options were clearly inferior to orthotopic HTX, most of these patients received no transplantation at all. Transplantation using hearts from donors with primary PHT for recipients with a high PVR (domino procedure) is another viable but rare option in transplant centers where patients undergo heart and lung transplantation for primary PHT. ${ }^{12}$

\section{THERAPY WITH A VENTRICULAR ASSIST DEVICE CAN REVERSE "FIXED” PHT}

As an alternative, reports and case studies have been published showing that pulsatile ventricular assist devices (VADs) reduce PHT in patients with end-stage heart failure. In 1991 Gallagher and colleagues ${ }^{13}$ demonstrated in 16 patients that mechanical support with a Novacor device (Novacor, Inc, Rueil-Malmaison, France) resulted in reduced PHT. This observation was confirmed by other authors in the following years. ${ }^{14-16}$

In the year 2000 we have started to implant left ventricular assist devices (LVAD) in patients with fixed PVR for destination therapy. A significant reduction in PVR was observed
TABLE 1. Pharmacologic testing for reversal of pulmonary vascular resistance before listing for heart transplantation: Freiburg protocol

1. Determination of baseline hemodynamics

2. Application of oxygen Lifeline mask $5 \mathrm{~L} / \mathrm{min}$

3. Application of nitrates

4. Application of inhalative iloprost

5. Application of dobutamine

6. Application of inhalative nitric oxide

$30-100 \mu \mathrm{g} / \mathrm{min}$

$2.5-5 \mu \mathrm{g}$

$2-6 \mu \mathrm{g} / \mathrm{kg} / \mathrm{min}$

$40 \mathrm{ppm}$ or oral application of sildenafil $25 \mathrm{mg}$

after several months of mechanical circulatory support. Eventually, the patients could be listed for HTX and underwent successful transplantation. After fixed PVR was successfully reversed with pulsatile and nonpulsatile VADs in the first 6 patients, this experience was published as a preliminary report in 2004. ${ }^{17}$ The reduction in PVR occurred even in patients with markedly elevated PVR ( $>7 \mathrm{WU}) .{ }^{17}$ Importantly, PHT did not return after HTX in our patients.

This observation that unloading of the left ventricle by pulsatile and nonpulsatile VADs is able to reverse previously "fixed" PVR was subsequently confirmed by other groups. ${ }^{3,6,18-21}$ Survival of these patients after transplantation was comparable with that of patients without prior PHT. ${ }^{22}$

Reversal of fixed PVR occurs after a short period (weeks to months) of mechanical circulatory support. Salzberg and coworkers $^{21}$ reported this decrease to occur after 6 weeks of LVAD support. This is confirmed by Etz and colleagues ${ }^{3}$ for the majority of their patients after implantation of nonpulsatile LVADs; after 6 months all their patients had PVR less than 3 WU. Zimpfer and associates ${ }^{6}$ also reported that the decrease in PVR occurred within a 6-week follow-up period.

Normalization of PVR after lowering the pressures in the left-sided heart chambers is not a recent observation in VAD

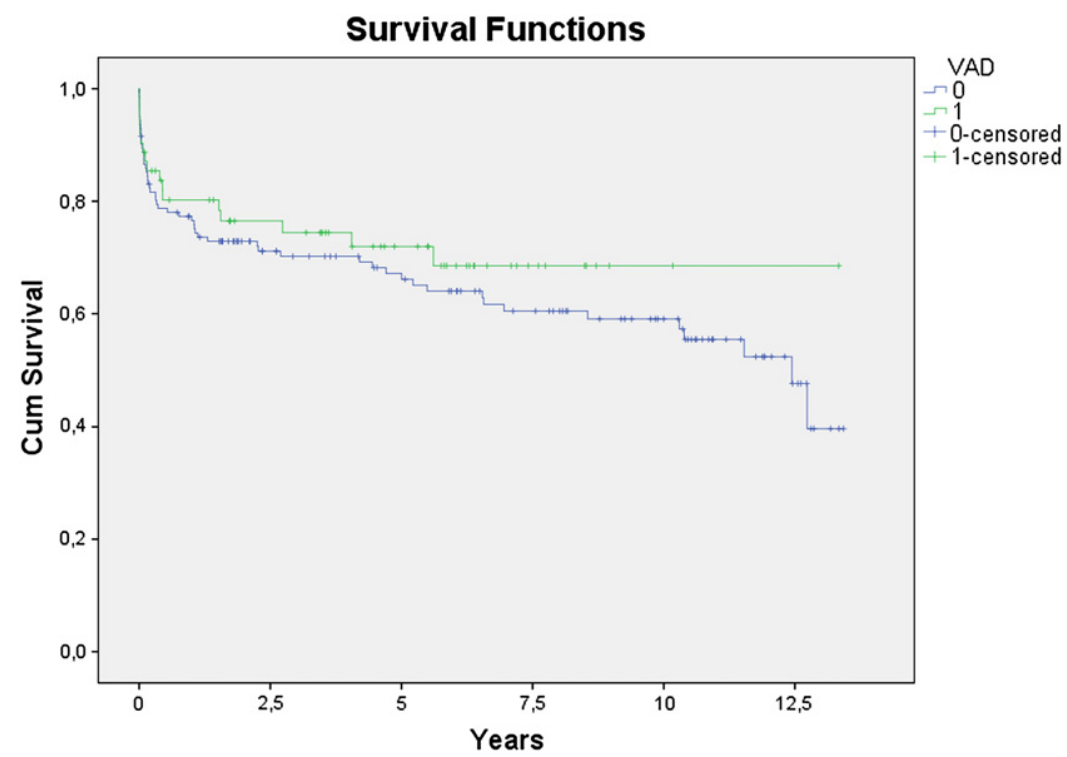

FIGURE 1. Long-term survival after heart transplantation in patients with and without prior mechanical circulatory support: Freiburg experience. $V A D$, Ventricular assist device. 
patients with congestive heart failure. There are also reports showing the same regression of PVR after successful orthotopic HTX in patients with elevated (reversible) PVR before HTX (normalization of PVR at 1 year after HTX). ${ }^{11}$ In addition, it has been known for decades that high PVR owing to severe mitral stenosis can also be reversed if pressures in the left atrium are decreased by mitral valve surgery. ${ }^{23}$

The pathophysiology of PHT in patients with terminal heart failure is a multifactorial process not finally elucidated, but mainly related to left ventricular failure. ${ }^{24}$ The mechanisms by which unloading of the left ventricle and atria (eg, by VAD) result in reversal of "fixed" PVR are unknown at present. One mechanism might be that relieved pulmonary congestion and normalized pulmonary hemodynamics reduce overproduction of intrinsic vasoconstrictors, which has been triggered by shear stress previously. ${ }^{3}$

Our own experience with mechanical circulatory support before HTX shows that the posttransplant survival is at least as good as (if not better than) that of patients receiving HTX without prior VAD support (Figure 1). These excellent longterm HTX results in patients with prior LVAD therapy as compared with those without prior LVAD support have also been reported by others. ${ }^{5}$

The major disadvantage of mechanical circulatory support is still the morbidity and mortality rate. However, recent publications have shown a significant improvement in the results after long-term mechanical support with 6- and 12-month survivals of $80 \%$ and $70 \%$, respectively. ${ }^{25}$

\section{CONCLUSIONS}

In conclusion, "fixed" PHT in patients with terminal heart failure can be reversed by temporary VAD support. This reduction in PVR occurs by 6 to 12 weeks in most patients, even in those with markedly elevated PVR (>7 WU). Long-term post-HTX outcome after reversal of "fixed" PHT with VADs is as good as (or even better than) that of HTX in patients without fixed PVR. However, VAD complications (morbidity and mortality) also have to be considered if this kind of treatment is chosen.

\section{References}

1. Cehn JM, Levin HR, Michler RE, Prusmack CJ, Rose EA, Aaronson KD. Reevaluating the significance of pulmonary hypertension before cardiac transplantation: determination of optimal thresholds and quantification of the effect of reversibility on perioperative mortality. J Thorac Cardiovasc Surg. 1997;114:627-34.

2. Erickson KW, Costanzo-Nordin MR, O'Sullivan EJ, Johnson MR, Zucker MJ, Pifarré R, et al. Influence of preoperative transpulmonary gradient on late mortality after orthotopic heart transplantation. J Heart Transplant. 1990;9:526-37.

3. Etz CD, Welp HA, Tjan TD, Hoffmeier A, Weigang E, Scheld HH, et al. Medically refractory pulmonary hypertension: treatment with nonpulsatile left ventricular assist devices. Ann Thorac Surg. 2007;83:1697-706.
4. Elefteriades JA, Lovoulos CJ, Tellides G, Goldstein LJ, Rocco EJ, Condos SG, et al. Right ventricle-sparing heart transplant: promising new technique for recipients with pulmonary hypertension. Ann Thorac Surg. 2000;69:1858-63.

5. Deng MC, Gradaus R, Hammel D, Weyand M, Günther F, Kerber S, et al. Heart transplant candidates at high risk can be identified at the time of initial evaluation. Transpl Int. 1996;9:38-45.

6. Zimpfer D, Zrunek P, Roethy W, Czerny M, Schima H, Huber L, et al. Left ventricular assist devices decrease fixed pulmonary hypertension in cardiac transplant candidates. J Thorac Cardiovasc Surg. 2007;133:689-95.

7. Nauser TD, Stites SW. Pulmonary hypertension: new perspectives. Congest Heart Fail. 2003;9:155-62.

8. Mehra MR, Kobashigawa J, Starling R, Russell S, Uber PA, Parameshwar J, et al. Listing criteria for heart transplantation: International Society for Heart and Lung Transplantation guidelines for the care of cardiac transplant candidates-2006. J Heart Lung Transplant. 2006;25:1024-42.

9. Ross H, Hendry P, Dipchand A, Giannetti N, Hirsch G, Isaac D, et al. 2001 Canadian Cardiovascular Society Consensus Conference on cardiac transplantation. Can J Cardiol. 2003;19:620-54.

10. Natale ME, Pina IL. Evaluation of pulmonary hypertension in heart transplant candidates. Curr Opin Cardiol. 2003;18:136-40.

11. Klotz S, Deng MC, Hanafy D, Schmid C, Stypmann J, Schmidt C, et al. Reversible pulmonary hypertension in heart transplant candidates-pretransplant evaluation and outcome after orthotopic heart transplantation. Eur J Heart Fail. 2003;5:645-53.

12. Birks EJ, Yacoub MH, Anyanwu A, Smith RR, Banner NR, Khaghani A. Transplantation using hearts from primary pulmonary hypertensive donors for recipients with high pulmonary vascular resistance. J Heart Lung Transplant. 2004; 23:1339-44.

13. Gallagher RC, Kormos RL, Gasior T, Murali S, Griffith BP, Hardesty RL. Univentricular support results in reduction of pulmonary resistance and improved right ventricular function. ASAIO Transplant. 1991;37:M287-8.

14. Smedira NG, Massad MG, Navia J, Vargo RL, Patel AN, Cook DJ, et al. Pulmonary hypertension is not a risk factor for RVAD use and death after left ventricular assist system support. ASAIO J. 1996;42:M733-5.

15. Baldovinos A, Kalangos A, Sierra J, Licker MJ, Faidutti B. Is partially reversible pulmonary hypertension a contraindication for heart transplantation? Transplant Proc. 2000;32:468-9.

16. Petrofski JA, Hoopes CW, Bashore TM, Russell SD, Milano CA. Mechanical ventricular support lowers pulmonary vascular resistance in a patient with congential heart disease. Ann Thorac Surg. 2003;75:1005-7.

17. Martin J, Siegenthaler MP, Friesewinkel O, Fader T, van de Loo A, Trummer G et al. Implantable left ventricular assist device for treatment of pulmonary hypertension in candidates for orthotopic heart transplantation - a preliminary study Eur J Cardiothorac Surg. 2004;25:971-7.

18. Al-Khaldi A, Ergina P, DeVarennes B, Lachappelle K, Cecere R. Left ventricular unloading in a patient with end-stage cardiomyopathy and medically unresponsive pulmonary hypertension. Artif Organs. 2004;28:158-60.

19. Choong CK, Pasque MK, Shelton K, Kehoe-Huck B, Ewald GA Horstmanshof D, et al. The beneficial role of left ventricular assist device destination therapy in the reversal of contraindications to cardiac transplantation. J Thorac Cardiovasc Surg. 2005;130:879-80.

20. Haddad H, Elabbassi W, Moustafa S, Davies R, Mesana T, Hendry P, et al. Left ventricular assist device as bridge to heart transplantation in congestive heart failure with pulmonary hypertension. ASAIO J. 2005;51:456-60.

21. Salzberg SP, Lachat ML, von Harbou K, Zund G, Turina M. Normalization of high pulmonary vascular resistance with LVAD support in heart transplantation candidates. Eur J Cardiothorac Surg. 2005;27:222-5.

22. Zimpfer D, Zrunek P, Sandner S, Schima H, Grimm M, Zuckermann A, et al. Posttransplant survival after lowering fixed pulmonary hypertension using left ventricular assist devices. Eur J Cardiothorac Surg. 2007;31:698-702.

23. Mears EJ, Harvey WP, Hufnagel CA. Relief of pulmonary hypertensive pain after mitral commissurotomy. N Engl J Med. 1953;249:715-8.

24. Rabinovitch M. Pulmonary hypertension: pathophysiology as a basis for clinical decision making. J Heart Lung Transplant. 1999;18:1041-53.

25. Slaughter MS, Rogers JG, Milano CA, Russell SD, Conte JV, Feldman D, et al. Advanced heart failure treated with continuous-flow left ventricular assist device. N Engl J Med. 2009;361:2241-51. 